\title{
Secure Enterprise and Read Performance Enhancement in Data Deduplication for Secondary Storage
}

\author{
S. Usharani, K. Kungumaraj
}

\begin{abstract}
With the tremendous growth of available digital data, the use of Cloud Service Providers (CSPs) are gaining more popularity, since these types of services promise to provide convenient and efficient storage services to end-users by taking advantage of a new set of benefits and savings offered by cloud technologies in terms of computational, storage, bandwidth, and transmission costs. we propose solutions for different data types (text, image and video) for secure data de-duplication in cloud environments. Our schemes allow users to upload their data in a secure and efficient manner such that neither a semi-honest CSP nor a malicious user can access or compromise the security of the data. Moreover, we propose proof of storage protocols including Proof of Retrievability (POR) and Proof of Ownership $(P O W)$ so that users of cloud storage services are able to ensure that their data has been saved in the cloud without tampering or manipulation. Experimental results are provided to validate the effectiveness of the proposed schemes. proposes a method to improve the read performance by investigating the recently accessed chunks and their locality in the backup set (data stream). Based on this study of the distribution of chunks in the data stream, few chunks are identified that need to be accumulated and stored to serve the future read requests better. This identification and accumulation happen on cached chunks. By this a small degree of duplication of the de-duplicated data is introduced, but by later caching them together during the restore of the same data stream, the read performance is improved. Finally the read performance results obtained through experiments with trace datasets are presented and analyzed to evaluate the design.
\end{abstract}

Keywords--- Cloud, Chunk Fragmentation, Significant, Recall, Queries.

\section{INTRODUCTION}

The secure enterprise data de-duplication exhibited in this Chapter is an instance of the plan proposed as in it centers around the structure of an enterprise demonstrate where it is accepted that distinctive enterprises running a similar sort of business can utilize a single cloud, and every one of these enterprises has its own internal clients. The clients belonging to a given enterprise store their data in the cloud using the enterprise server. A two-levels data de-duplication conspire is introduced: one at the enterprise level, and the other at the CSP level. At the enterprise level, every individual enterprise plays out its own data de-duplication among its clients (cross-client de-duplication). At the CSP level, a second data de-duplication is performed on the data presented by the diverse enterprises to the cloud (crossenterprise data de-duplication). As far as anyone is concerned, no earlier work has been done that bargains with ensuring the security of data de-duplication in the cloud using an enterprise display where cross client and crossenterprise data de-duplication are both integrated. The value of the proposed model is that it tends to be helpful for little or medium size enterprises that don't have an immense number of assets and that intend to utilize these assets for other internal calculations or tasks rather than for capacity reason.

The main structure highlights of our proposed enterprise model can be condensed as pursues: Enterprise Level Data De-duplication: We propose an answer for the enterprises that can help maximizing their capacity savings in the cloud. This arrangement applies the previously mentioned twolevel de-duplication procedures, to be specific single and cross-client data de-duplication at the enterprise level, trailed by cross-enterprise data de-duplication at the cloud stockpiling supplier level. Secure Indexing Scheme: B* Tree-Based Secure Indexing Scheme: Certain record qualities are utilized for indexing, with the objective to boost the de-duplication proportions. The configurations of the tree index and the data index are planned so that data deduplication is upheld at both the enterprise and CSP levels. In addition, a focalized encryption system is likewise utilized during the time spent indexing, which is a basic piece of the data de-duplication process.

Despite the fact that the write process is basic with respect to storage framework performance (write performance), the read process is similarly critical. Reestablish speed straightforwardly impacts the RTO of the framework. Those frameworks which deal with basic data like that of income division, defense segment, and so on. can't manage the cost of longer downtimes. This implies the significance of read performance. The framework talked about in this work expects to improve this read performance by making copy duplicates of a portion of the de-copied chunks amid read process. This is finished by investigating the situation of chunks in the data stream that is in effect right now read back and stored in the read reserve. The presence of a chunk in a reinforcement form of a data stream with respect to its territory in the as of late gotten to chunk succession is concentrated to decide upon whether it ought to be copied or not. Henceforth, the read store utilized is called 'Reminiscent Read Cache'. It is additionally accepted that the read succession of data is equivalent to the write grouping.

Manuscript received September 16, 2019.

S. Usharani, Research Scholar, Department of Computer Science, Mother Teresa Women's University, Kodaikanal, Tamil Nadu, India.

K. Kungumaraj, Assistant Professor, PG Department of Computer Science, Arulmigu Palaniandavar Arts College for Women, Palani, Tamil Nadu, India. 


\section{SECURE ENTERPRISE AND READ PERFORMANCE ENHANCEMENT IN DATA DEDUPLICATION FOR SECONDARY STORAGE}

Dominant part of the earlier works are identified with write performance improvement subsequently proficiently detecting and expelling whatever number copies as could be expected under the circumstances with the assistance of productive variable-sized square data tending to, index enhancement, and data compartment design. Nam et al. initially proposed a marker esteem for degraded read performance, called Chunk Fragmentation Level (CFL). Afterward, in they proposed a way to deal with improve the read performance utilizing the CFL esteem. These three strategies are connected amid write process along these lines degrading the write performance. though in this work, the strategy proposed is connected amid the read process and dependent on different elements.

\section{LITERATURE SURVEY}

[1] Chi Yang and Jinjun Chen proposed a novel scalable data compression based on similarity calculation among the partitioned data chunks with Cloud computing. A similarity model was developed to generate the standard data chunks for compressing big data sets. Instead of compression over basic data units, the compression was conducted over partitioned data chunks. The MapReduce programming model was adopted for the algorithms implementation to achieve some extra scalability on Cloud. With the real meteorological big sensing data experiments on our U-Cloud platform, it was demonstrated that our proposed scalable compression based on data chunk similarity significantly improved data compression performance gains with affordable data accuracy loss. The significant compression ratio brought dramatic space and time cost savings. With the popularity of Spark and its specialty in processing streaming big data set. [2] Youjip Won, Kyeongyeol Lim, and Jaehong Min proposed a novel multicore chunking algorithm, MUCH, which parallelizes the variable size chunking. To date, most of the existing works on deduplication focus on expediting the redundancy detection process, while less attention has been paid on how to make the file chunking faster. That proposed a multicore chunking algorithm, $\mathrm{MUCH}$, which guarantees Chunking Invariability. They developed a performance model to compute the segment size that maximizes the chunking bandwidth while minimizing the memory requirement. Through extensive physical experiments, we showed that the performance of MUCH scales linearly with the number of cores. In quad-core CPUs, MUCH brings a 400 percent performance increase when the storage device is sufficiently fast. The benefits of MUCH are evident when it chunks large files, e.g., tar images of file system snapshot, at high performance storage. MUCH successfully increases the chunking performance with the factor being as high as the number of available CPU cores without any additional hardware assistance. [3] Xu Zhang and Yue Cao propose a fully distributed ICN-based caching scheme for content objects in Radio Access Network (RAN) at eNodeBs. Such caching scheme operates in a cooperative way within neighbourhoods, aiming to reduce cache redundancy so as to decision logic at individual eNodeBs allows for adaptive caching, by taking into account dynamic context information, such as content popularity and availability. The improve the diversity of content distribution. The caching

efficiency of the proposed distributed caching scheme is evaluated via extensive simulations, which show great performance gains, in terms of a substantial reduction of backhaul content traffic as well as great improvement on the diversity of content distribution, etc. [4] Chuanshuai Yu, Chengwei Zhang, Yiping Mao, Fulu Li presented the leapbased CDC algorithm and added a secondary condition to it in order to reduce the computing overhead and maintain the same deduplication ratio. This algorithm satisfies both the content defined condition and the equal probability condition. The leap-based CDC algorithm with or without a secondary condition can significantly reduce the computing overhead while maintaining the same deduplication ratio. To resolve the technique issue of not being able to use the rolling hash in the new algorithm, they introduced the pseudo-random transformation to replace the role of rolling hash. [5] Daniel Posch, Hermann Hellwagner and Peter Schartner proposed a framework for multimedia delivery in VoD use cases. The concepts of CCN, DASH and BE in order to create dynamic adaptive encrypted chunks of data, which can be inherently cached in the network. The evaluation results show that network inherent caching can definitely increase the efficiency of multimedia delivery. However, the usage of adaptive concepts leads to the question of how to synchronize clients to exploit the advantage of cached data perfectly. Finding a solution to this issue would enhance the framework greatly.

\section{METHODOLOGY}

\subsection{Proposed Load Balancing and Local-Global Source De-duplication}

The proposed distribute hash table (DHT ) based burden adjusting pattern and the proposed Application-aware Local Global source (ALG) blueprint for de-duplication construction is spurred in close to home cloud registering condition is designed to meet the necessity of burden adjusting and de-duplication proficiency with high deduplication viability and low framework overhead. The primary idea of the proposed ALG Dedupeis 1) misusing both low-overhead local resources and high-overhead cloud resources to lessen the computational overhead by utilizing a canny data chunking plan and a versatile utilization of hash capacities dependent on application awareness, and 2) to alleviate the on circle index query bottleneck by separating the full index into little independent and application-explicit files in an application-aware index structure. 3) The fundamental idea of the proposed DHT based burden adjusting diagram is to play out the heap adjusting undertaking for put away documents in the ALG Dedupe stage, where the proposed strategy the errand are proficiently adjusted. The capacity nodes are organized as a system dependent on distributed hash tables (DHTs), e.g., finding a record chunk can essentially allude to fast key query in DHTs, given that a one of a kind handle (or identifier) is allocated to each document chunk.

Published By: 
The proposed outcomes improve the detection aftereffects of the data excess records with low framework overhead on the customer side and profoundly adjusted framework result in the individual cloud figuring condition. A design diagram of proposed ALG and DHT is represented in Figure 1, where minor documents are first sifted through by record measure channel for productivity reasons, and reinforcement data streams are broken into chunks by a savvy chunker utilizing an application aware chunking procedure. Data chunks from a similar kind of records are then de-copied in the application aware de-duplicator by creating chunk fingerprints in hash motor and performing data repetition check in application-aware lists in both local customer and remote cloud. DHTs empower nodes to self-arrange and Repair while continually offering query usefulness in node dynamism, streamlining the framework arrangement and the executives. The chunk servers in our proposition are sorted out as a DHT arrange.

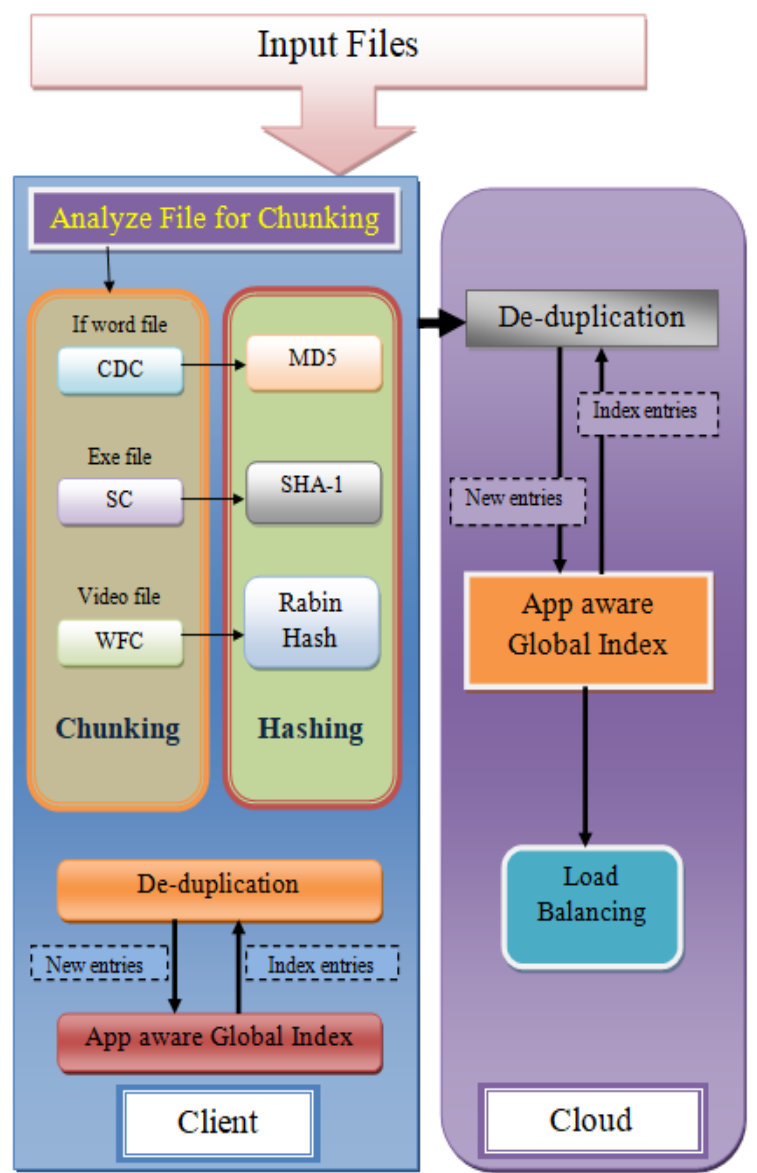

Figure 1: Architectural overview of the proposed ALG de-duplication and DHT for load balancing design

In distributed file frameworks (e.g., Google GFS and Hadoop HDFS), a consistent number of imitations for each file chunk are kept up in particular nodes to improve file accessibility as for node disappointments and departures. Our present burden adjusting calculation does not treat reproductions particularly. It is far-fetched that at least two imitations are set in an identical node due to the arbitrary idea of our heap rebalancing calculation. All the more explicitly, each under loaded node tests various nodes, each chose with a likelihood of $1 / n$, to share their heaps (where $n$ is the all out number of capacity nodes).

\section{PROPOSED WORK}

Phase 1: Secure Enterprise Data De-duplication in the Cloud

We are proposing a scheme for private data deduplication convention in the cloud stockpiling setting. We might want to feature the critical specialized contrast among open and private data de-duplication conventions. In private data de-duplication conventions, we consider the data to be encoded by the client first before the transfer, with keys not being imparted to the cloud provider, though openly data deduplication conventions the data is either uploaded either in plain content form or scrambled with shared keys among clients and the cloud stockpiling provider. In the former, for example private data de-duplication conventions, the effect of encryption on clients' data in various can make crossenterprise de-duplication endeavors challenging.

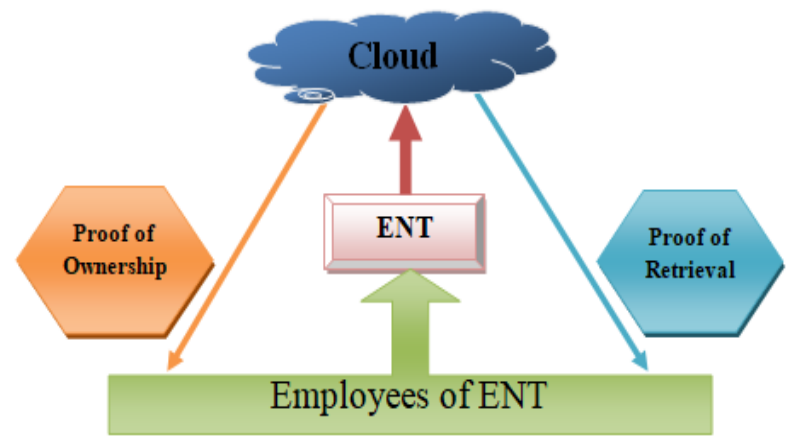

\section{Figure 2: Flow of POR and POW Protocols among the} Cloud, ENT and Users

The workers are the clients of the cloud stockpiling administrations and are approved to run the POR convention with the cloud for assuring the integrity of the data in the cloud. These clients will likewise be scrutinized by the cloud for the ownership of their data through the POW convention. Figure: 2 depicts the operational stream for POR and POW conventions involving all the three players. There are a few essential ways to deal with check the integrity of put away data in the cloud. A guileless methodology is for the client to download the file and check its integrity. In most application, this speaks to pricey methodology regarding bandwidth use and thus it doesn't speak to a practical alternative. Another basic methodology requires a client to register a keyed hash $\mathrm{hk}(\mathrm{F})$ for a given key $\mathrm{k}$ and a file $\mathrm{F}$. The client would then transfer the source file $F$ to the cloud, while retaining the hash esteem $\mathrm{hk}(\mathrm{F})$. For the POR, the client would send the key used to the cloud stockpiling provider and request that it figure and resend the hash of the file. By keeping many hash esteems and their corresponding keys, the client can run this POR convention various number of times. There are a few downsides to this methodology including the requirement for the cloud stockpiling provider to figure the hash estimation of the whole file and the linear connection between's the quantity of the keys and the hash esteems kept to the quantity of POR inquiries which can be make.

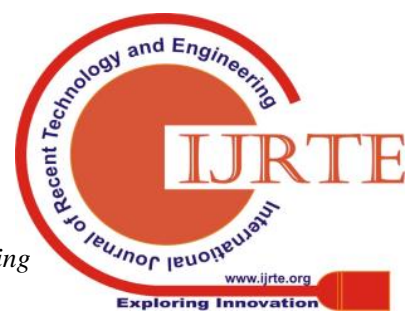




\section{SECURE ENTERPRISE AND READ PERFORMANCE ENHANCEMENT IN DATA DEDUPLICATION FOR SECONDARY STORAGE}

In the following area, we will exhibit an efficient and secure scheme for a POR convention in which a client can confirm the integrity of his data against the cloud stockpiling provider who is practicing data de-duplication at the enterprise level.

\section{Phase 2: Read Performance Enhancement in Data De- duplication for Secondary Storage}

\section{ProposedScheme-Reminiscent ReadCache}

Reminiscent Read Cache model deals with just single data stream for read/write. Above all, it includes altering the LRU store into one that likewise recalls the chunks in the reserved holders that were gotten to previously. In view of this, a minor degree of duplication is presented by copying and gathering utilized chunks from under-used reserved holders, in a cradle in the memory. These chunks are really copies of already read chunks in the data stream chunk succession. The support is called Dupe Buffer and is of fixed size, equivalent to that of an ordinary measured holder. The copied chunks called Dupe Chunks, are aggregated in a holder in this support called the Dupe Container which is intermittently flushed to plates dependent on the nearness of its ID in a Past Access Window, limiting it to various sizes. By this variable-sized Dupe Containers are created in the framework. Four distinctive realized sizes are utilized, to be specific, quarter, half, seventy five percent and full estimated holders where full size is that of a customary measured compartment. Strangely, the Dupe Buffer can likewise be considered to be a piece of the store and can serve reserve hits.

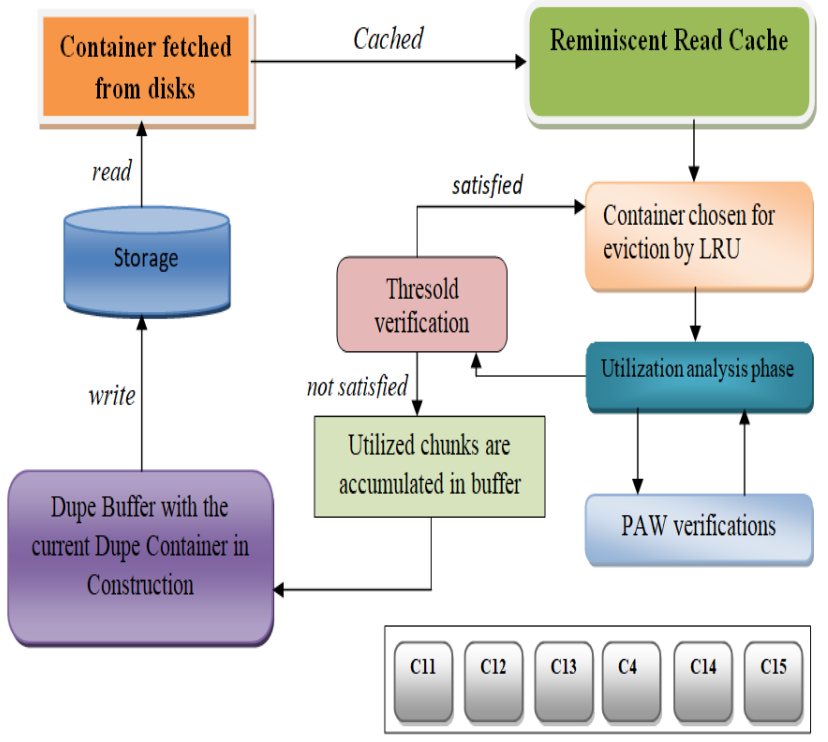

Past Access Window(PAW) with Container IDs

Figure 3: Reminiscent ReadCache Framework

\section{EXPERIMENTAL RESULTS}

Average Similarity Measure

Table 1: Average Similarity Measure

\begin{tabular}{|l|l|l|l|}
\hline Existing 1 & Existing 2 & Existing 3 & Proposed \\
\hline $\mathbf{1 7 . 3}$ & 13.54 & 40 & 62.54 \\
\hline $\mathbf{2 5}$ & 18.97 & 59.66 & 80 \\
\hline $\mathbf{3 0 . 6}$ & 27.71 & 62.34 & 93.6 \\
\hline $\mathbf{4 8 . 9 6}$ & 38.02 & 76.9 & 109.1 \\
\hline $\mathbf{5 0 . 3 2}$ & 46.66 & 83.45 & 123.42 \\
\hline
\end{tabular}

The comparison table of average similarity measure is demonstrates the different value if existing and proposed method.

While comparing the existing and proposed method the proposed method values are better than the existing method.

Existing 1 value starts from 17.3 to 50.32 existing 2 value starts from 13.54 to 46.66 existing 3 values starts from 40 to 83.45 and proposed values starts from 62.54 to 123.42.Every time the proposed method gives the great results.

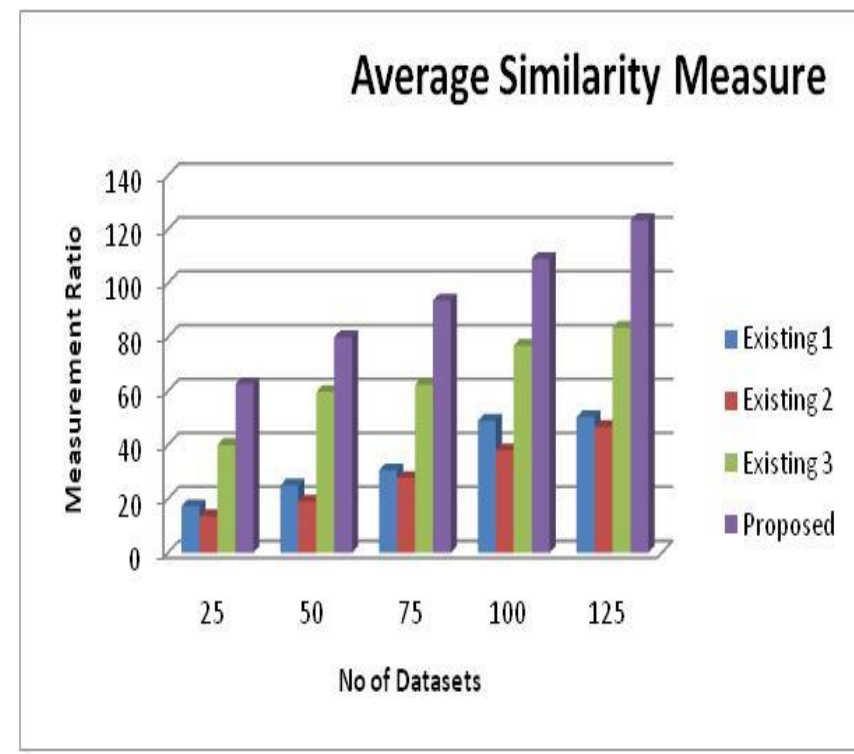

Figure 4: Average Similarity Measure

The comparison chart of Average Similarity Measure is demonstrates the existing and proposed method values.No of datasets in $\mathrm{x}$ axis and measurement ratio in $\mathrm{y}$ axis. Proposed method demonstrates the better results than the existing method.

Existing 1 value starts from 17.3 to 50.32 existing 2 value starts from 13.54 to 46.66 existing 3 values starts from 40 to 83.45 and proposed values starts from 62.54 to 123.42 .

\section{Average Recall}

Table 2: Average Recall

\begin{tabular}{|l|l|l|l|}
\hline Existing 1 & Existing 2 & Existing 3 & Proposed \\
\hline $\mathbf{2 7 . 3}$ & 10.54 & 50 & 76.54 \\
\hline $\mathbf{4 5}$ & 28.97 & 69.66 & 100 \\
\hline $\mathbf{7 0 . 6}$ & 37.71 & 82.34 & 123.6 \\
\hline $\mathbf{8 8 . 9 6}$ & 48.02 & 96.9 & 149.1 \\
\hline $\mathbf{1 1 0 . 3 2}$ & 66.66 & 123.45 & 173.42 \\
\hline
\end{tabular}

The comparison table of average recall is demonstrates the different value if existing and proposed method.while comparing the existing and proposed method the proposed method values are better than the existing method. Existing 1 value starts from 27.3 to 110.32 existing 2 value starts from 10.54 to 66.66 existing 3 values starts from 50 to 123.45 and proposed values starts from 76.54 to 173.42.Every time the proposed method gives the great results. 


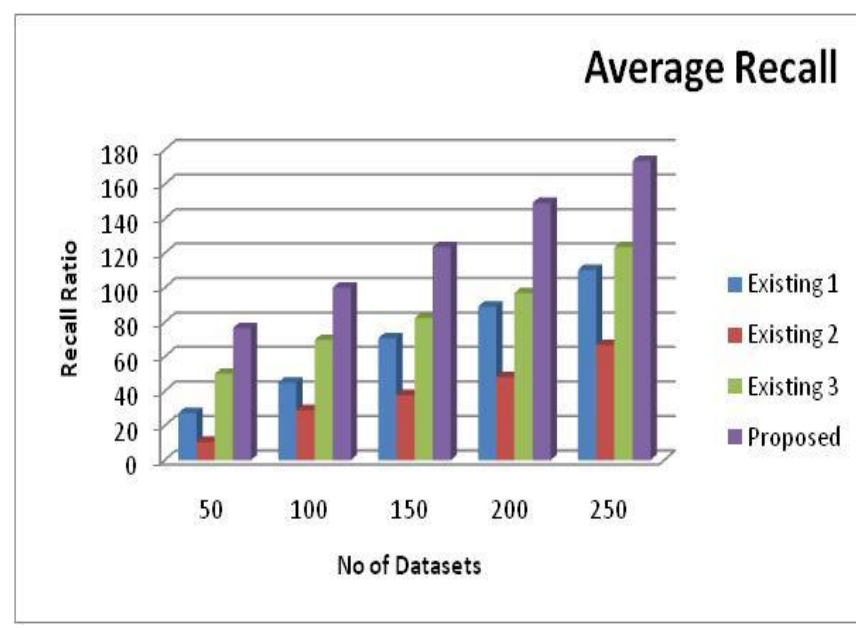

Figure 5: Average Recall

The comparison chart of Average Recall demonstrates the existing and proposed method values. No of datasets in $x$ axis and measurement ratio in $y$ axis. Proposed method demonstrates the better results than the existing method. Existing 1 value starts from 27.3 to 110.32 existing 2 value starts from 10.54 to 66.66 existing 3 values starts from 50 to 123.45 and proposed values starts from 76.54 to 173.42

\section{$\%$ Queries}

Table 3: \%Queries

\begin{tabular}{|l|l|l|l|}
\hline Existing 1 & Existing 2 & Existing 3 & Proposed \\
\hline $\mathbf{1 7 . 8 9}$ & 13.67 & 8.99 & 19.1 \\
\hline $\mathbf{2 3 . 3 3}$ & 19.89 & 10.87 & 25.65 \\
\hline $\mathbf{2 8 . 6}$ & 24.4 & 13.26 & 30 \\
\hline $\mathbf{3 2 . 6 7}$ & 28.97 & 18.77 & 35.45 \\
\hline $\mathbf{3 5 . 8 9}$ & 32.45 & 25.1 & 39.99 \\
\hline
\end{tabular}

The comparison table of average \%Queries is demonstrates the different value if existing and proposed method.while comparing the existing and proposed method the proposed method values are better than the existing method. Existing 1 value starts from 17.89 to 35.89 existing 2 value starts from 13.67 to 32.45 existing 3 values starts from 8.99 to 25.1 and proposed values starts from 19.1 to 39.99.Every time the proposed method gives the great results.

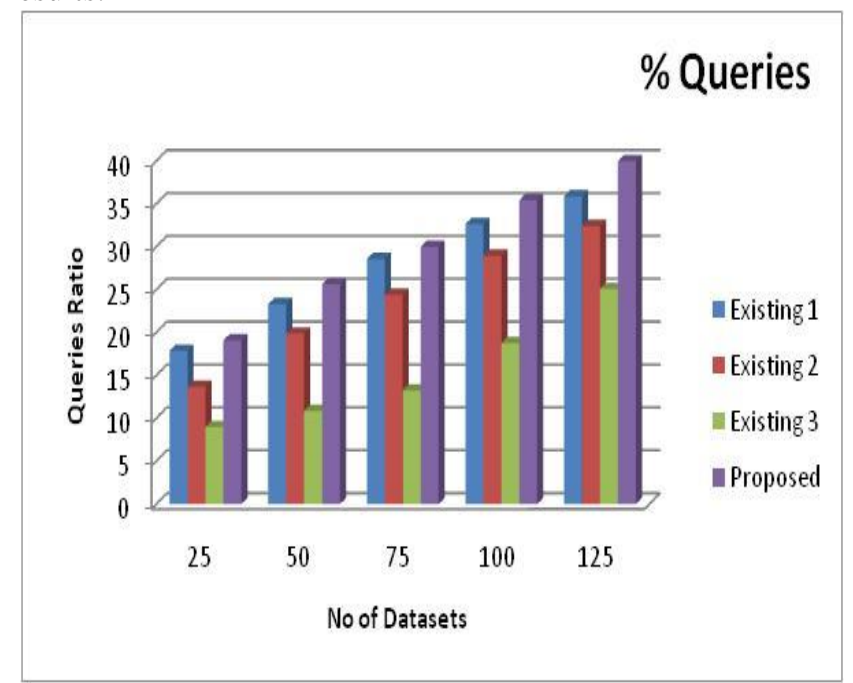

Figure 6:\% Queries

The comparison chart of Average Recall demonstrates the existing and proposed method values. No of datasets in $\mathrm{x}$ axis and queries ratio in $y$ axis. Proposed method demonstrates the better results than the existing method. Existing 1 value starts from 17.89 to 35.89 existing 2 value starts from 13.67 to 32.45 existing 3 values starts from 8.99 to 25.1 and proposed values starts from 19.1 to 39.99 .

\section{CONCLUSION}

Proposed a two-level data de-duplication structure that can be utilized in the cloud stockpiling by enterprises who share a single regular CSP for their administrations. By employing the cross-client data de-duplication performed at the enterprise level and the cross enterprise data deduplication performed at the CSP level, it is normal that enterprises can re-appropriate their data to the cloud while the CSP can accomplish cost and space savings. The system is designed dependent on the constraint that the CSP is semi-genuine, thereby can't be believed when handling clients' data. The proposed plan was at last approved with tests which demonstrated to deliver better read performance in data de-duplication than the standard plan. To additionally improve this framework, future research has been arranged where best in class and modified pre-getting and reserving systems will be utilized when scaled to bigger datasets with bigger store. This will likewise help in dealing with numerous data streams simultaneously. With numerous data streams each stream requires to have its own dedicated store space. Additionally, copies crosswise over data streams will require the store spaces to know about each other in a worldwide namespace. Chunk fragmentation will have increasingly antagonistic impacts with numerous simultaneous data streams than a solitary stream. The limit esteem determination for least compartment usage is right now static. This will be made versatile in future. Likewise, depending on the remaining burden this esteem will shift so framework delivers the ideal and anticipated performance. Outstanding task at hand portrayal ends up basic in these situations. Without causing overhead the outstanding task at hand will be checked and concentrated to settle on decisions on the limit. The framework will have an input module which can help in the versatile idea of the edge.

\section{REFERENCES}

1. Chi Yang and Jinjun Chen, "A Scalable Data Chunk Similarity based Compression Approach for Efficient Big Sensing Data Processing on Cloud", Published in: IEEE Transactions on Knowledge and Data Engineering (Volume: 29, Issue: 6, June 1 2017), Publisher: IEEE, Date of Publication: 18 February 2016, Print ISSN: 1041-4347; Electronic ISSN: 1558-2191; CD-ROM ISSN: 2326-3865

2. Youjip Won, Kyeongyeol Lim, and Jaehong Min, "MUCH: Multithreaded Content-Based File Chunking", Published in: IEEE Transactions on Computers ( Volume: 64, Issue: 5, May 12015 ), Publisher: IEEE; Date of Publication: 14 May 2014; Date of Publication: 14 May 2014

3. Xu Zhang and Yue Cao, "A Cooperation-Driven ICNbased Caching Scheme for Mobile Content Chunk Delivery at RAN", Published in: 2017 13th International Wireless Communications and Mobile Computing

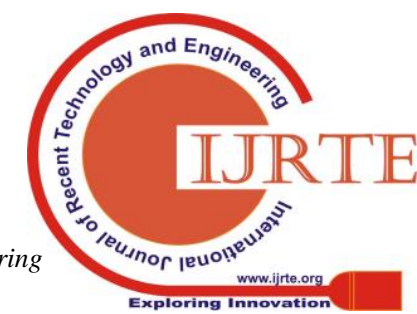


Conference (IWCMC); Publisher: IEEE; Electronic ISSN: 2376-6506

4. Chuanshuai Yu, Chengwei Zhang, Yiping Mao, Fulu Li, "Leap-based Content Defined Chunking --- Theory and Implementation", Published in: 2015 31st Symposium on Mass Storage Systems and Technologies (MSST); Publisher: IEEE; Print ISSN: 2160-195X; Electronic ISSN: $2160-1968$

5. Daniel Posch, Hermann Hellwagner and Peter Schartner, "On-Demand Video Streaming based on Dynamic Adaptive Encrypted Content Chunks", Published in: 2013 21st IEEE International Conference on Network Protocols (ICNP); Publisher: IEEE; Electronic ISBN: 978-1-4799-1270-4; Print ISSN: 1092-1648

6. Chi Yang and Jinjun Chen, "A Scalable Data Chunk Similarity based Compression Approach for Efficient Big Sensing Data Processing on Cloud", Published in: IEEE Transactions on Knowledge and Data Engineering ( Volume: 29, Issue: 6, June 12017 ); Publisher: IEEE; Print ISSN: 1041-4347; Electronic ISSN: 1558-2191; CD-ROM ISSN: 2326-3865

7. C. Goktug Gurler, S. Sedef Savas, and A. Murat Tekalp, "VARIABLE CHUNK SIZE AND ADAPTIVE SCHEDULING WINDOW FOR P2P STREAMING OF SCALABLE VIDEO”, Published in: 2012 19th IEEE International Conference on Image Processing; Publisher: IEEE; Print ISSN: 1522-4880; Online ISSN: 1522-4880; Electronic ISSN: 2381-8549

8. Haiying Shen and Jin Li , "A DHT-Aided Chunk-Driven Overlay for Scalable and Efficient Peer-to-Peer Live Streaming", Published in: IEEE Transactions on Parallel and Distributed Systems ( Volume: 24, Issue: 11, Nov. 2013 ); Publisher: IEEE; Date of Publication: 22 October 2012; Print ISSN: 1045-9219; Electronic ISSN: 15582183; CD-ROM ISSN: 2161-9883

9. Deepavali Bhagwat, Kave Eshghi, Darrell D. E. Long and Mark Lillibridge, "Extreme Binning: Scalable, Parallel Deduplication for Chunk-based File Backup", Published in: 2009 IEEE International Symposium on Modeling, Analysis \& Simulation of Computer and Telecommunication Systems; Publisher: IEEE; Print ISBN: 978-1-4244-4927-9; CD-ROM ISBN: 978-14244-4928-6

10. Chu-Hsing Lin, Chen-Yu Lee, Yi-Shiung Yeh, HungSheng Chien and Shih-Pei Chien, "Generalized Secure Hash Algorithm: SHA-X”, Published in: 2011 IEEE EUROCON - International Conference on Computer as a Tool; Publisher: IEEE; Electronic ISBN: 978-1-42447487-5; Print ISBN: 978-1-4244-7486-8;CD-ROM ISBN: 978-1-4244-7485-1

11. Sang-Hyun Lee, Kyung-Wook Shin, "An Efficient Implementation of SHA processor Including Three Hash Algorithms (SHA-512, SHA-512/224, SHA-512/256)", Published in: 2018 International Conference on Electronics, Information, and Communication (ICEIC); Publisher: IEEE; Print on Demand(PoD) ISBN: 978-15386-4754-7

12. IMTIAZ AHMAD AND A. SHOBA DAS, "Analysis and Detection Of Errors In Implementation Of SHA-512 Algorithms On FPGAs", Published in: The Computer Journal ( Volume: 50, Issue: 6, Nov. 2007 ); Publisher: IEEE; Date of Publication: Nov. 2007; Print ISSN: 00104620; Electronic ISSN: 1460-2067

13. Alavi Kunhu, Hussain Al-Ahmad and Fatma Taher, "Medical Images Protection and Authentication using hybrid DWT-DCT and SHA256-MD5 Hash Functions", Published in: 2017 24th IEEE International Conference on Electronics, Circuits and Systems (ICECS); Publisher: IEEE; Electronic ISBN: 978-1-5386-1911-7; Print on Demand(PoD) ISBN: 978-1-5386-1912-4
14. Mochamad Vicky Ghani Aziz, Rifki Wijaya, Ary Setijadi Prihatmanto, Diotra Henriyan, "HASH MD5 Function Implementation at 8-bit Microcontroller", Published in: 2013 Joint International Conference on Rural Information \& Communication Technology and ElectricVehicle Technology (rICT \& ICeV-T); Publisher: IEEE; Electronic ISBN: 978-1-4799-3365-5; Print ISBN: 9781-4799-3363-1;CD-ROM ISBN: 978-1-4799-336

15. Eko Sediyono, Kartika Imam Santoso and Suhartono, "Secure Login by Using One-time Password Authentication Based on MD5 Hash Encrypted SMS", Published in: 2013 International Conference on Advances in Computing, Communications and Informatics (ICACCI), Publisher: IEEE; Electronic ISBN: 978-1-4673-6217-7; Print ISBN: 978-1-47992432-5; CD-ROM ISBN: 978-1-4799-2659-6 